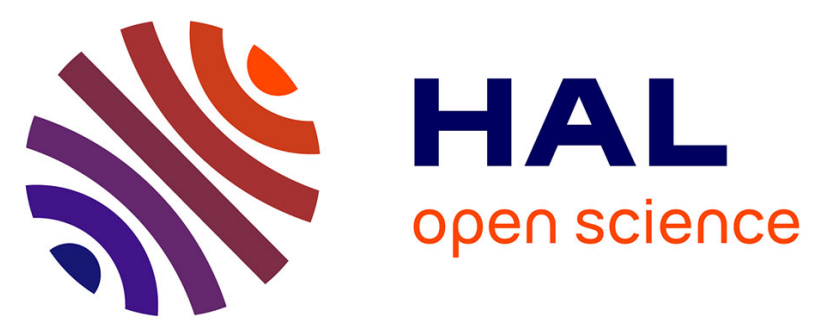

\title{
MULTI-PHYSICS MODELLING CONTRIBUTIONS TO INVESTIGATE THE ATMOSPHERIC COSMIC RAYS ON THE SINGLE EVENT UPSET SENSITIVITY ALONG THE SCALING TREND OF CMOS TECHNOLOGIES
}

\author{
G Hubert, D Regis, A Cheminet, M Gatti, V Lacoste
}

\section{To cite this version:}

G Hubert, D Regis, A Cheminet, M Gatti, V Lacoste. MULTI-PHYSICS MODELLING CONTRIBUTIONS TO INVESTIGATE THE ATMOSPHERIC COSMIC RAYS ON THE SINGLE EVENT UPSET SENSITIVITY ALONG THE SCALING TREND OF CMOS TECHNOLOGIES. Radiation Protection Dosimetry, 2014, 161 (1-4), pp.290-294. 10.1093/rpd/ncu003 . hal-01082948

\author{
HAL Id: hal-01082948 \\ https://hal.science/hal-01082948
}

Submitted on 14 Nov 2014

HAL is a multi-disciplinary open access archive for the deposit and dissemination of scientific research documents, whether they are published or not. The documents may come from teaching and research institutions in France or abroad, or from public or private research centers.
L'archive ouverte pluridisciplinaire HAL, est destinée au dépôt et à la diffusion de documents scientifiques de niveau recherche, publiés ou non, émanant des établissements d'enseignement et de recherche français ou étrangers, des laboratoires publics ou privés. 


\title{
MULTI-PHYSICS MODELLING CONTRIBUTIONS TO INVESTIGATE THE ATMOSPHERIC COSMIC RAYS ON THE SINGLE EVENT UPSET SENSITIVITY ALONG THE SCALING TREND OF CMOS TECHNOLOGIES
}

\author{
G. Hubert ${ }^{1, *}$, D. Regis ${ }^{2}$, A. Cheminet ${ }^{1,3}$, M. Gatti ${ }^{2}$ and V. Lacoste ${ }^{2}$ \\ ${ }^{1}$ The French Aerospace Lab, ONERA, Toulouse 31055, France \\ ${ }^{2}$ Thales Systèmes Aéroportés, Pessac 33600, France \\ ${ }^{3}$ IRSN, Saint Paul-Lez-Durance 13115, France \\ *Corresponding author: guillaume.hubert@onera.fr
}

\begin{abstract}
Particles originating from primary cosmic radiation, which hit the Earth's atmosphere give rise to a complex field of secondary particles. These particles include neutrons, protons, muons, pions, etc. Since the 1980s it has been known that terrestrial cosmic rays can penetrate the natural shielding of buildings, equipment and circuit package and induce soft errors in integrated circuits. Recently, research has shown that commercial static random access memories are now so small and sufficiently sensitive that single event upsets (SEUs) may be induced from the electronic stopping of a proton. With continued advancements in process size, this downward trend in sensitivity is expected to continue. Then, muon soft errors have been predicted for nano-electronics. This paper describes the effects in the specific cases such as neutron-, proton- and muon-induced SEU observed in complementary metal-oxide semiconductor. The results will allow investigating the technology node sensitivity along the scaling trend.
\end{abstract}

\section{INTRODUCTION}

When the primary cosmic rays penetrate the Earth's atmosphere, they interact with atmospheric nuclei and induce nuclear reactions generating secondary radiation of every kind ${ }^{(1,2)}$. Products of the cosmic-ray showers are protons, electrons, neutrons, heavy ions, muons and pions. Secondary radiation and its intensity depend on the altitude, the geomagnetic latitude and the Sun's activity. Neutrons are measurable at $330-\mathrm{km}$ altitude, and their density increases with decreasing altitude until they reach a peak at $\sim 20 \mathrm{~km}$. At a sea level, muons ${ }^{(3)}$ are the most numerous terrestrial species. The muons are decay products of mesons produced in hadronic cascades initiated by primary cosmic rays, usually made of very energetic protons.

Radiation-induced single event effects (SEEs) have been identified as a major reliability issue for commercial electronic system ${ }^{(1)}$. Various types of SEEs are discussed in the literature and these effects can be separated into two main classes: soft errors and hard errors. Soft errors are modifications of the content of memory cells. They can be single bit upset (SBU) or multiple cell upset (MCU). The single event upset (SEU) includes SBU and MCU events. For energetic particles, nowadays, soft errors can induce an error rate higher than the sum of all other reliability concerns.

With transistors' dimensions continuously shrinking, more and more effects due to SEE appear in electronic devices. Among the encountered problems, those created by radioactive impurities [alpha-single event rate (SER)] and cosmic radiation are significant.
The constant evolution of micro-electronic circuits to nano-scale techniques makes the sensitivity thresholds of circuits being an increasing criticism against external aggressions. As the dependence of society with respect to electronic technology seems irreversible, it dependability becomes an essential element of the design.

The technological integration induces a set of mechanisms such as the charge-sharing effects, the drift and ambipolar diffusion, or the bipolar amplification. Moreover, new SEE problematic emerge consequently to technological integration as the radial profile of deposited charge induced by ions which can be comparable to the topology of cells ${ }^{(4)}$. With continued advancements in process manufacturing technology, the sensitivity to SEE is expected to increase and recent researches have demonstrated the occurrence of soft errors due to protons ${ }^{(5,6)}$ and muons ${ }^{(7)}$. Thus, this paper describes the effects in the specific cases such as neutron-, proton- and muon-induced SEU observed in complementary metal-oxide semiconductor (CMOS). The results will allow investigating the technology node sensitivity along the scaling trend of CMOS and will show that proton and muon environments will be critical to avionic and terrestrial applications, respectively.

\section{SEE MULTI-PHYSICS MODELLING}

Between the incident particle and the SEE occurrence, many physical mechanisms intervene: the particle crosses the structure, shielding and the package 


\section{G. HUBERT ET AL.}

before reaching the sensitive structure. Then, primary or secondary ions generate electron-hole pairs along their paths in the semiconductor. The pairs evolve into the media according to transport mechanisms and are collected by the electrodes of the device. Multi-level physical approaches are needed to model SEE for nano-scale devices.

\section{Multi-physics modelling based-on MUSCA SEP3}

MUSCA SEP $3^{(8)}$, firstly presented in 2009, consists in modelling the whole device within its local and global environments, and the detailed characteristics of the radiation field environment (nature, direction and spectrum). The results presented in 2009 have shown that each physical level is critical for SEE risk calculation including the environment description. In the framework of this study, MUSCA SEP3 is used to model respectively the CMOS technologies, their SEU sensitivities and the atmospheric radiation environments. Figure 1 describes MUSCA SEP3 platform which allows calculating the SEU sensitivity and characteristics.

\section{Atmospheric radiation modelling}

The first step consists in modelling the atmospheric neutron, proton and muon environments. Then, neutron, muon and proton spectra were calculated due to the excel-based program for calculating atmospheric cosmic-ray spectrum $\left(\right.$ EXPACS $\left.^{(9)}\right)$ developed by the Japan's Atomic Energy Agency. The neutron/ proton direction properties can be taking into account in modelling owing to $\operatorname{QARM}^{(10)}$ calculations, which provide downward and upward spectra. Concerning muons, the angular distribution is usually approximated to be $\cos ^{2}(\theta)^{(3)}$, where $\theta$ is the zenith angle.

At a sea level there are many thermal and epithermal neutrons (depending on the local environment). However, their contributions to SEU contribution are not significant. Manufacturers avoid using some atomic species in devices, such as the boron because of the high cross section of the reaction ${ }^{10} \mathrm{~B}\left(\mathrm{n}_{\mathrm{th}}, \alpha\right)^{7} \mathrm{Li}$.

Figure $2 \mathrm{a}$ and $\mathrm{b}$ presents, respectively, the spectra calculated for the ground and avionic altitudes, considering a location in Toulouse (France) and solar minimum solar conditions. It is not required to consider energies $<1 \mathrm{MeV}$. Indeed, muons and protons cannot reach the sensitive zone of the device because they lose their energies in the structure, package, metallisation, etc.

\section{Neutron, proton, muon and $\alpha$-SER modelling}

To model the interaction processes, MUSCA SEP3 integrates nuclear databases ${ }^{(8)}$ which provide secondary ions count, atomic and mass numbers, their energies and directions. Nuclear databases have been developed using GEANT4 $4^{(11)}$ for proton and neutron interactions with atomic species such as $\mathrm{Si}, \mathrm{O}, \mathrm{Cu}$ and $\mathrm{W}$ (main atomic species used in electronic devices) and in the $1-\mathrm{MeV}-2 \mathrm{GeV}$ energy range ${ }^{(8)}$.

A second database has been generated with SRIM (based on the Bethe-Block equation) that contains the linear energy transfer (LET) and range characteristics for proton-uranium/muon projectiles and several target materials $(\mathrm{Si}, \mathrm{W}, \mathrm{O}$ and $\mathrm{Cu})$. Muons cause

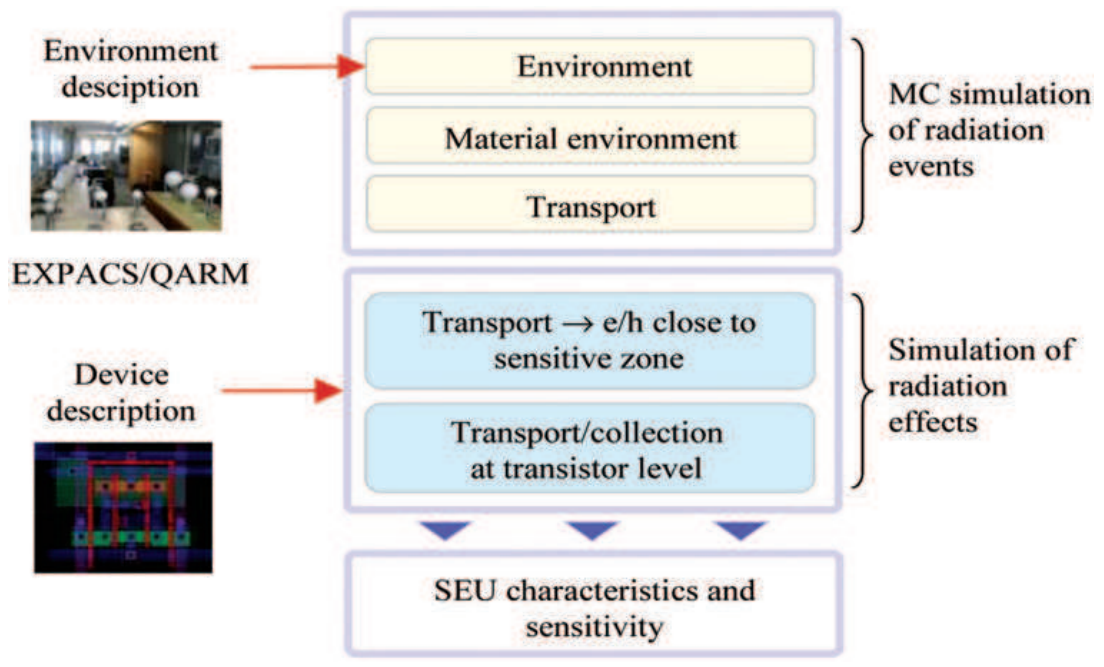

Figure 1 Global methodology applied in this work and based on MUSCA SEP3 platform. 
(a)

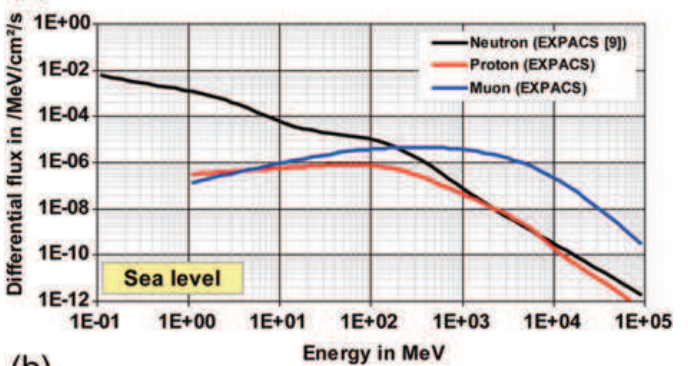

(b)

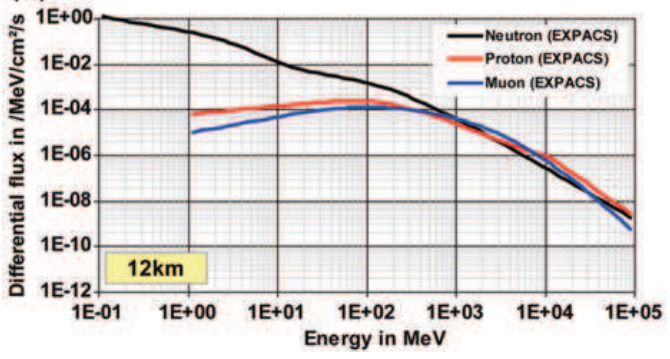

Figure 2 Neutron, proton and muon spectra issued from EXPACS $^{(9)}$ considering the (a) sea-level altitude and (b) avionic altitude $(12 \mathrm{~km})$.

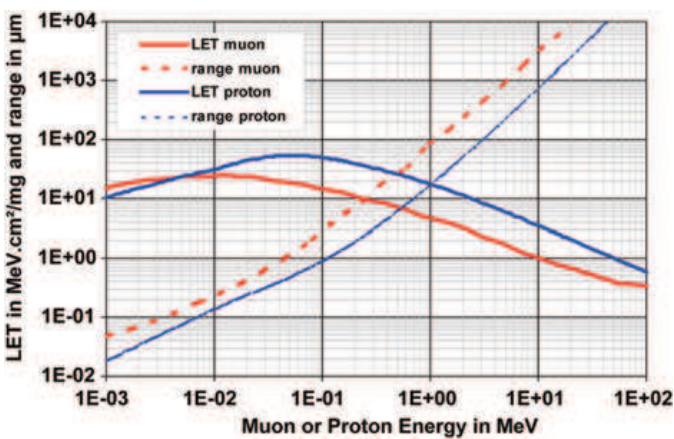

Figure 3 LET and range in silicon for protons and muons in the energy range of $1 \mathrm{keV}-100 \mathrm{MeV}$.

effects only through direct ionisation and Figure 3 shows the muon and proton ranges and LET in silicon as a function of energy. For terrestrial applications, alpha emitters are the main contributor to SER. Alpha-emitting impurities can be found in some packaging materials, chemicals and materials used in the fabrication process. The emission rate can strongly vary depending on the quantity and purification grade of these materials. Therefore, MUSCA SEP3 has been adapted to investigate and quantify the $\alpha$-SER contribution.
Table 1. Technological parameters including elementary cell surface, critical charge and power supply.

\begin{tabular}{lccc}
\hline $\begin{array}{l}\text { Node } \\
(\mathrm{nm})\end{array}$ & \multicolumn{3}{c}{ Technological parameters } \\
\cline { 2 - 4 } & $\begin{array}{c}\text { Cell surface } \\
\left(\mu \mathrm{m}^{2}\right)\end{array}$ & $\begin{array}{c}\text { Critical charge } \\
(\mathrm{fC})\end{array}$ & $\begin{array}{c}\text { Power supply } \\
(\mathrm{V})\end{array}$ \\
\hline 65 & 0.62 & 0.6 & 0.9 \\
45 & 0.5 & 0.4 & 0.85 \\
32 & 0.34 & 0.3 & 0.8 \\
28 & 0.23 & 0.2 & 0.7 \\
22 & 0.15 & 0.15 & 0.7 \\
14 & 0.1 & 0.1 & 0.5 \\
\hline
\end{tabular}

Thus, MUSCA SEP3 allows modelling the particle penetration and effect in device. It is possible to deduce the SER due to the following:

$$
\mathrm{SER}=\iint_{4 \pi E} \sigma(E, \vec{v}) \cdot \frac{\mathrm{d} \varphi(E, \vec{v})}{\mathrm{d} E \cdot \mathrm{d} \vec{v}} \cdot \mathrm{d} E \cdot \mathrm{d} \vec{v}
$$

where $\sigma(E, \vec{v})$ and $\mathrm{d} \varphi(E, \vec{v}) / \mathrm{d} E \cdot \mathrm{d} \vec{v}$ are, respectively, the SEU cross section and the energetic differential flux and the directional properties.

\section{Technological roadmap modelling}

Scaling is too often thought of as only reducing the geometric feature size of a transistor. Indeed, technology scaling may also include new materials, oxide changes, material resistance and new transistor architecture. The device description requires technological inputs such as the topology, layout and dimensions of the active areas. It is necessary to take into account many geometrical features such as shallow trench isolation (STI).

Then, MUSCA SEP3 is associated with a structure extraction methodology based on ITRS analyses or GDS general design specification) extractor which allows deducing information directly from layout files in GDS format such as the surfaces and positions of the drain electrodes for $\mathrm{N}$ type metal oxide semiconductor (NMOS) and $\mathrm{P}$ type metal oxide semiconductor (PMOS) transistors, N-well dimensions, cell spacing, STI, etc.

MUSCA SEP3 allows calculating the collected charge induced by ions for each zone likely to collect charge. The comparison between the collected charge and a critical charge $Q_{\text {crit }}$ which characterises the technology sensitivity allows determining the event occurrence and estimating the device sensitivity. Technological trend, which is characterised by lower operating voltages, lower nodal capacitance and higher integration density, induces an SEU and MCU increase.

Thus, Table 1 describes the main technological parameters used in models, including the cell size, the critical charge and the power supply. 


\section{RESULTS AND DISCUSSION}

The objective consists in evaluating the SEU trends in bulk considering 65-14 technological nodes against ground and avionic environments including neutron, proton, muon and $\alpha$-SER contributions. Figures 4 and 5 present the SER in failure in time (the number of failure per $10^{9} \mathrm{~h}$ ) as a function of technological scale for avionic and sea-level altitudes. The results show that the downscaling induces increased SEU susceptibility to radiation.

The results allow analysing the event multiplicity. The majority of events are characterised by MCU and multiplicities increase with the technological integration. MCU characterised by $>5$ bit events is the main contribution to SER for 22- and 14-nm devices.

\section{Avionic altitude}

For the avionic altitude, neutron and proton environments induce the main contribution to the total SER; however, the muon environment and $\alpha$-SER impact are negligible. Classically, the proton direct ionisation

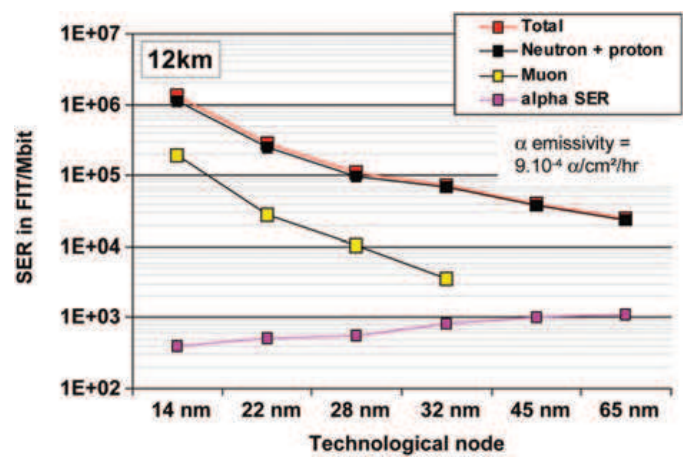

Figure 4 SER as a function of integration at an avionic altitude.

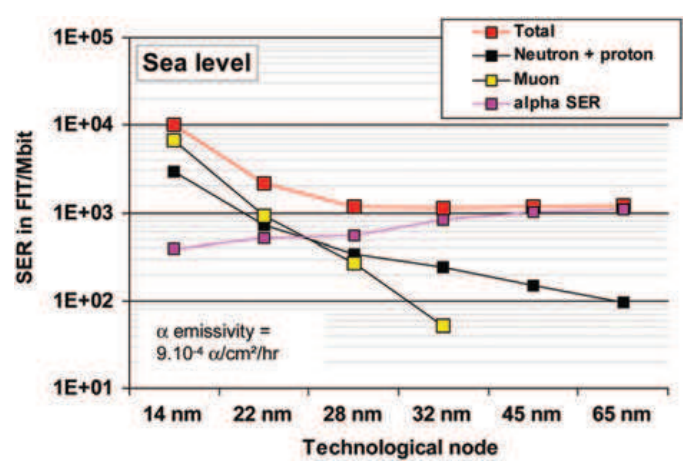

Figure 5 SER as a function of integration at a sea-level altitude. is not considered as a source of SEE for avionic applications. But, complementary analyses based on the results show that the proton environment impact (i.e. direct ionisation impact) is not negligible from $65 \mathrm{~nm}$ and it becomes the main contribution from $28 \mathrm{~nm}$ and beyond. Previous works emphasise that the technological integration for over $90 \mathrm{~nm}$ induces an SER saturation or even slight reduction coupled with an increase in the multiple event rates. However, accounting for the direct proton ionisation contribution leads to an expected strong increase in the SER from $28 \mathrm{~nm}$ and beyond.

\section{Ground altitude}

$\alpha$-SER is the main contribution down to $28 \mathrm{~nm}$ for the ground altitude. The $\alpha$-SER contribution is more important compared with the neutron, proton and muon contributions. Calculations consider a ultralow alpha (i.e. $\epsilon<10^{-3} \alpha \mathrm{cm}^{-2} \cdot \mathrm{h}^{-1}$ ) package (order of magnitude consistent with underground experiments $\left.^{(12)}\right)$. Thus, it is clear that the direct ionisation of proton and muon SEU sensitivities is not a critical problematic for ground applications to date. Moreover, results suggest muon-induced upset do affect the soft error rate from the 32-nm SRAM operated at a nominal supply voltage, and they have a significant impact on circuits fabricated in smaller process technologies $(22$ and $14 \mathrm{~nm})$. In addition, the results show that muon impact becomes the main contribution from $22 \mathrm{~nm}$ and beyond.

To induce an SEU by direct ionisation, the Bragg peak must be localised in the sensitive region of the device. Therefore, accounting the local and global environments of the target in its operational configuration (structure, package and device including metallisation, passivation and semiconductor layers) only a specific energy range is able to meet this condition. Considering muon direction properties, the angle of incidence and location of strike are also critical parameters to induce an SEU with this mechanism. Thus, analyses allow showing that the low-energy muon spectrum $(\sim 10 \mathrm{MeV})$ is responsible for SEU.

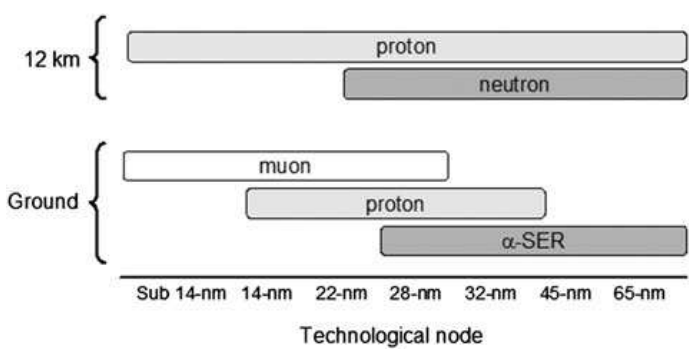

Figure 6 Risk assessment applied to scaling CMOS trend for avionic $(12 \mathrm{~km})$ and ground environments. 


\section{CONCLUSIONS}

This paper proposes to model and investigate the atmospheric cosmic rays impact on the SER along the scaling trend. Neutron, proton and muon environments are considered for two operational altitudes, i.e. avionic and at sea level.

A synthesis about the SEU/risk assessment applied to scaling CMOS trend for avionic and ground environments can be proposed due to these investigations (Figure 6).

First results indicate that protons/neutrons must be taken into account for avionic environments while muons will be critical for terrestrial applications. Moreover, at a ground level SRAM but also flip flop and combinational logic based on nanometric technologies may become sensitive to the low-energy muon spectrum. Then, future terrestrial error rate predictions will require characterisation of the device LET threshold, consideration of the muon environment and advanced radiation transport computations.

\section{REFERENCES}

1. Ziegler, J. F. Terrestrial cosmic rays. IBM J. Res. Dev. 40(1), 19-39 (1996).

2. Barth, J. L. et al. Space, atmospheric, and terrestrial radiation environments. IEEE Trans. Nucl. Sci. 50(3), 466482 (2003).
3. Nagamine, K. Chapter 3-Muon Inside Condensed Matter: Introductory Muon Science. Cambridge University Press (2003).

4. Raine, M. et al. Impact of the radial ionization profile on SEE prediction for SOI transistors and SRAMs beyond the 32-nm technological node. IEEE Trans. Nucl. Sci. 58(3), 840-847 (2011).

5. Sierawski, B. D. et al. Impact of low-energy proton induced upsets on test methods and rate predictions. IEEE Trans. Nucl. Sci. 56(6), 3085-3092 (2009).

6. Hubert, G. et al. MUSCA SEP3 contributions to investigate the direct ionization proton upset in $65 \mathrm{~nm}$ technology for space, atmospheric and ground applications. In: RADECS, Brugge, Belgium (2009).

7. Sierawski, B. D. et al. Muon-induced single event upsets in deep-submicron technology. IEEE Trans. Nucl. Sci. 57(6), 3273-3278 (2010).

8. Hubert, G. et al. Operational SER calculations on the $S A C$-C orbit using the multi-scales single event phenomena predictive platform (MUSCA SEP3). IEEE Trans. Nucl. Sci. 56(6), 3032-3042 (2009).

9. EXPACS Homepage. Available on http://phits.jaea.go. jp/expacs (last accessed on January 2014).

10. QinetiQ Atmospheric Radiation Model (QARM) [online]. Available on http://www.qarm.eu (last accessed on January 2014).

11. GEANT4 Homepage. Available on http://geant4.web. cern.ch (last accessed on January 2014).

12. Lesea, A. et al. Qualification methodology for submicron ICs at the low noise underground laboratory of Rustrel. IEEE Trans. Nucl. Sci. 55(4), 2148-2153 (2008). 